\title{
SOLUTION OF THE STRESS-EQUILIBRIUM AND COMPATABILITY EQUATIONS IN THE PRESENCE OF BODY FORCES AND ARBITRARY TEMPERATURE FIELDS*
}

\author{
BY \\ DONALD R. CHILDS \\ General Dynamics Corporation, Quincy, Mass.
}

\begin{abstract}
We have solved the stress-equilibrium and compatibility equations in three dimensions in terms of a scalar stress function which satisfies a fourth order partial differential equation. The solution also includes operations on two integrals which exhibit the longitudinal and shear components explicitly. All possible relationships involving equilibrium and compatibility conditions result in the same fourth order differential equation involving time and space coordinates. For the static case this equation reduces to the biharmonic equation $\nabla^{4} \chi=0$.

1. Introduction. Up to the present time it has been considered difficult, if not impossible to solve the three dimensional stress equilibrium and compatibility equations in terms of a scaler stress function which satisfies a biharmonic equation, except under very special circumstances. Timoshenko ${ }^{1}$ has given the static stress components of a circular cylinder in the absence of body forces and temperature field in terms of a scalar stress function which satisfies a biharmonic equation.

However, if one starts out with Hooke's Law, the equilibrium condition, and the compatibility conditions, one can derive the dynamic equilibrium and compatibility equations in the presence of body forces and temperature field and define a displacement vector for small displacement theory. The displacement vector enables one to derive Navier's equation.

It is shown in the text that a solution of Navier's equation guarantees a solution to the equilibrium and compatibility equations since the stresses are derived from the Duhamel-Neumann relations. Navier's equation is separated into a homogeneous and an inhomogeneous part, the inhomogeneous solution being the solution to the $\mathrm{d}^{\prime}$ Alembert wave equation. The homogeneous solution is obtained in terms of a single scalar stress function which satisfies a fourth order equation. In the static case, the fourth order equation reduces to the biharmonic equation.

We use the Lamé constants $\nu, \lambda, E$, and $G$ defined by

$$
\begin{aligned}
& E=2 G(1+\nu)=\text { Young's Modulus, } \\
& \nu=\frac{\lambda}{2(\lambda+G)}=\text { Poisson's Ratio, }
\end{aligned}
$$

$G$ being the shear modulus.

It should be noted that our solution reduces to Timoshenko's solution and biharmonic equation for a cylinder for the static case in the absence of body force and a temperature field.

*Received February 17, 1967.

${ }^{1} \mathrm{~S}$. Timoshenko and J. N. Goodier, Theory of elasticity, Second Edition, McGraw-Hill, New York, 1951, pp. 343. Also see Sneddon, Handbuch der Physik, VI, P. 105. 
2. Equilibrium and compatibility equations. We start from the Duhamel-Neumann relations

$$
\epsilon_{i i}=\frac{1}{2 G} \sigma_{i i}+\left(\alpha T-\frac{\nu S}{E}\right) \delta_{i i}
$$

where, $\alpha$ is the coefficient of linear expansion

$T$ the temperature,

and where $\delta_{i j}=0$ if $i \neq j$ and 1 if $i=j$ and $S=\sum_{i=i}^{3} \sigma_{i i}$.

If we take the trace of the strain tensor, we find that

$$
\sum_{i=i}^{3} \epsilon_{i j}=e=3 \alpha T+\frac{1-2 v}{E} S .
$$

Solving for $S$ and replacing it in Eq. (2.1) we have

$$
\sigma_{i j}=2 G \epsilon_{i j}+[\lambda e-(3 \lambda+2 G) \alpha T] \delta_{i j} .
$$

The compatibility conditions require that

$$
\frac{\partial^{2} \epsilon_{i j}}{\partial x_{k} \partial x_{l}}+\frac{\partial^{2} \epsilon_{k l}}{\partial x_{i} \partial x_{i}}-\frac{\partial^{2} \epsilon_{i k}}{\partial x_{i} \partial x_{l}}-\frac{\partial^{2} \epsilon_{i l}}{\partial x_{i} \partial x_{k}}=0
$$

for $i, j, k, l=1,2,3$. These equations are satisfied exactly if

$$
\epsilon_{i j}=\frac{1}{2}\left(\partial u_{i} / \partial x_{i}+\partial u_{i} / \partial x_{i}\right) .
$$

One can see then that $e=\nabla \cdot \mathbf{u}$ and

$$
\sigma_{i j}=G \partial u_{i} / \partial x_{i}+G \partial u_{i} / \partial x_{i}+[\lambda \nabla \cdot \mathbf{u}-(3 \lambda+2 G) \alpha T] \delta_{i j} .
$$

The equilibrium condition is

$$
\sum_{j} \partial \sigma_{i j} / \partial x_{i}+K_{i}=0
$$

where it is understood that $K_{i}=F_{i}-\rho\left(\partial^{2} u_{i} / \partial t^{2}\right)$ where $F$ is the body force.

By manipulating the compatibility conditions (Eq. 2.4) and making use of (Eq. 2.7), one can derive the compatibility equations,

$$
\begin{aligned}
\nabla^{2} \sigma_{i j}+\frac{1}{1+\nu} \frac{\partial^{2} S}{\partial x_{i} \partial x_{i}}+\frac{\alpha E}{1+\nu}\left(\frac{\partial^{2} T}{\partial x_{i} \partial x_{i}}\right. & \left.+\frac{1+\nu}{1-\nu} \delta_{i j} \nabla^{2} T\right) \\
& +\frac{\nu}{1-\nu} \delta_{i j} \nabla \cdot K+\frac{\partial K_{i}}{\partial x_{i}}+\frac{\partial K_{i}}{\partial x_{i}}=0 .
\end{aligned}
$$

Making use of the definition of $K$, we have

$$
\sum_{i} \partial \sigma_{i j} / \partial x_{i}+F_{i}=\rho\left(\partial^{2} u_{i} / \partial t^{2}\right)
$$

and

$$
\begin{aligned}
\nabla^{2} \sigma_{i j}+\frac{1}{1+\nu} & \frac{\partial^{2} S}{\partial x_{i} \partial x_{i}}+\frac{\alpha E}{1+\nu}\left(\frac{\partial^{2} T}{\partial x_{i} \partial x_{i}}+\frac{1+\nu}{1-\nu} \delta_{i j} \nabla^{2} T\right) \\
& +\frac{\nu}{1-\nu} \delta_{i j} \nabla \cdot F+\frac{\partial F_{i}}{\partial x_{i}}+\frac{\partial F_{i}}{\partial x_{i}}=\frac{\rho \nu}{1-\nu} \delta_{i j} \frac{\partial^{2} e}{\partial t^{2}}+2 \rho \frac{\partial^{2} \epsilon_{i j}}{\partial t^{2}}
\end{aligned}
$$


It will be convenient to calculate the solutions in Cartesian form. One can obtain the stress components for another geometry by transforming each of the components, or one can change the tensor form of the solutions into dyadic form, thus allowing one to write out the component of any geometry immediately. We shall do this in the appendix and exhibit the cylindrical and spherical components explicitly.

We can rewrite the equilibrium and compatibility equations in terms of the displacements. They both are equivalent and are made up of a homogeneous part and an inhomogeneous part. The inhomogenous portion of the equation comes from the contribution of the body forces and temperature field. The inhomogeneous part leads to a particular integral which can be obtained by solving the wave equation.

The homogeneous portion has as its solution arbitrary functions which can be determined by the boundary conditions imposed on the displacements and/or the stresses. Since the displacements and stress components are derived from the same stress function, they can be used in any combination as boundary conditions which are consistent with the equilibrium and compatibility conditions.

3. Solution to equations. Let us write the equilibrium equation (2.10) in terms of the displacements. We obtain

$$
(\lambda+G) \frac{\partial}{\partial x_{i}}(\nabla \cdot u)+G \nabla^{2} u_{i}-(3 \lambda+2 G) \alpha \frac{\partial^{\prime} T}{\partial x_{i}}+F_{i}-\rho \frac{\partial^{2} u_{i}}{\partial t^{2}}=0 .
$$

Making use of the definition of the Lamé constants, we obtain

$$
\frac{1}{1-2 \nu} \frac{\partial}{\partial x_{i}} \nabla \cdot u+\nabla^{2} u_{i}-\frac{2(1+\nu)}{1-2 \nu} \alpha \frac{\partial T}{\partial x_{i}}+\frac{1}{G} F_{i}-\frac{\rho}{G} \frac{\partial^{2} u_{i}}{\partial t^{2}}=0 .
$$

If we rewrite the compatibility equation (2.11) in terms of the displacement and manipulate, we obtain

$$
\begin{aligned}
& \frac{\partial}{\partial x_{i}}\left[G \nabla^{2} u_{i}+\frac{G}{1-2 \nu} \frac{\partial}{\partial x_{i}} \nabla \cdot u-2 \alpha G \frac{(1+\nu)}{1-2 \nu} \frac{\partial T}{\partial x_{i}}+F_{i}-\rho \frac{\partial^{2} u_{i}}{\partial t^{2}}\right] \\
& +\frac{\partial}{\partial x_{i}}\left[G \nabla^{2} u_{i}+\frac{G}{1-2 \nu} \frac{\partial}{\partial x_{i}} \nabla \cdot u-2 \alpha G \frac{(1+\nu)}{1-2 \nu} \frac{\partial T}{\partial x_{i}}+F_{i}-\rho \frac{\partial^{2} u_{i}}{\partial t^{2}}\right]+\frac{2 \nu G}{1-2 \nu} \\
& \cdot \delta_{i j}\left[\nabla^{2} \nabla \cdot u-\frac{1+\nu}{1-\nu} \alpha \nabla^{2} T+\frac{1-2 \nu}{2 G(1-\nu)} \nabla \cdot F-\rho \frac{(1-2 \nu)}{2 G(1-\nu)} \frac{\partial^{2}}{\partial t^{2}} \nabla \cdot u\right]=0 .
\end{aligned}
$$

The terms in the first two brackets of Eq. (3.3) are the same as the terms in Eq. (3.2), and the terms in the third bracket represent the divergence of Eq. (3.2). Thus a solution to Eq. (3.2) is automatically a solution to Eq. (3.3).

Let us rewrite Eq. (3.2) in the form

$$
\frac{1}{1-2 \nu} \nabla \nabla \cdot u+\nabla^{2} u-\frac{\rho}{G} \frac{\partial^{2} u}{\partial t^{2}}=\frac{2(1+\nu)}{1-2 \nu} \alpha \nabla T-\frac{1}{G} F .
$$

The homogeneous portion of the equation is

$$
\frac{1}{1-2 \nu} \nabla \nabla \cdot u+\nabla^{2} u-\frac{\rho}{G} \frac{\partial^{2} u}{\partial t^{2}}=0 .
$$

The static equivalent of Eq. (3.5) is

$$
(1 /(1-2 v)) \nabla \nabla \cdot u_{1}+\nabla^{2} u_{1}=0 .
$$


In order for $\nabla \nabla \cdot u$ and $\nabla^{2} u$ to be of the same form, we should choose

$$
u_{1}=a(\hat{n} \cdot \nabla) \nabla \chi_{1}+b \hat{n} \nabla^{2} \chi_{1} .
$$

The operator $(\hat{n} \cdot \nabla)$ is understood to be $(\partial / \partial x+\partial / \partial y+\partial / \partial z)$ and $n$ is the vector $(1,1,1)$. We can choose $a$ and $b$ so that all terms of the form $(\hat{n} \cdot \nabla) \nabla \chi$ cancel.

$$
\text { If } b=-2(1-\nu) a \text {, then from Eq. (3.6) } \nabla^{4} \chi_{1}=0 \text {. }
$$

In the time dependent case, we should choose $u$ in the form

$$
u=a(\hat{n} \cdot \nabla) \nabla \chi+b \hat{n} \nabla^{2} \chi+c \hat{n}\left(\partial^{2} \chi / \partial t^{2}\right)
$$

Again we wish to choose $a, b$, and $c$ such that all terms containing $(\hat{n} \cdot \nabla) \nabla \chi$ or $(\hat{n} \cdot \nabla) \nabla\left(\partial^{2} \chi / \partial t^{2}\right)$ cancel. If $b=-2(1-\nu) a$ and $c=(1-2 \nu)(\rho / G) a$, then Eq. (3.5) leads to the equation

$$
(1-\nu) \nabla^{4} \chi-\frac{\rho}{2 G}(3-4 \nu) \frac{\partial^{2}}{\partial t^{2}} \nabla^{2} \chi+\frac{\rho^{2}}{2 G^{2}}(1-2 \nu) \frac{\partial^{4} \chi}{\partial t^{2}}=0
$$

Rather than choose the axis of symmetry to be along the vector $(1,1,1)$ we could just as easily have chosen this axis to be one of the coordinate axis, say (0.0.1). The $z$ axis is usually chosen by convention to be the axis of symmetry of a body of revolution. Thus we can choose $u$ in the form

$$
u=a \frac{\partial}{\partial z} \nabla \chi+b \hat{k} \nabla^{2} \chi+c \hat{k} \frac{\partial^{2} \chi}{\partial t^{2}}
$$

i.e.,

$$
u_{x}=a \frac{\partial^{2} \chi}{\partial z \partial x}, \quad u_{y}=a \frac{\partial^{2} \chi}{\partial z \partial y}, \quad u_{z}=a \frac{\partial^{2} \chi}{\partial z^{2}}+b \nabla^{2} \chi+c \frac{\partial^{2} \chi}{\partial t^{2}} .
$$

with the same relations between $a, b$, and $c$ as given above. Because the axis of symmetry and the derivative with respect to the axis of symmetry commutes with the gradient and the Laplacian, Eq. (3.11) is a valid definition of $u$ and it will satisfy Eq. (3.5) provided that the scalar stress function obeys Eq. (3.10).

If there are body forces and temperature fields present, we need to find the particular integral for Eq. (3.4). Since every vector is the sum of a gradient of a scalar and the curl of another vector, we can write

$$
u_{p}=\nabla \varphi+\nabla x \psi \text { and } F=-\nabla V+\nabla x f
$$

Equation (3.4) takes the form

$$
\nabla^{2} \nabla \varphi-\frac{\rho(1-2 \nu)}{2 G(1-\nu)} \frac{\partial^{2}}{\partial t^{2}} \nabla \varphi=\frac{1+\nu}{1-\nu} \alpha \nabla T+\frac{1-2 \nu}{2 G(1-\nu)} \nabla V
$$

and

$$
\nabla^{2}(\nabla x \psi)-\frac{\rho}{G} \frac{\partial^{2}}{\partial t^{2}}(\nabla x \psi)=-\frac{1}{G} \nabla x f
$$

Equation (3.14) has the solution 


$$
\begin{aligned}
\nabla \varphi=-\frac{1}{4 \pi} \frac{1+\nu}{1-\nu} \alpha \int_{V^{\prime}} \frac{\nabla^{\prime} T^{\prime}\left(\mathbf{r}^{\prime}, t-\frac{\left|\mathbf{r}-\mathbf{r}^{\prime}\right|}{a_{1}}\right)}{\left|\mathbf{r}-\mathbf{r}^{\prime}\right|} d V^{\prime} \\
\quad-\frac{1}{4 \pi} \frac{1-2 \nu}{2 G(1-\nu)} \int_{V^{\prime}} \frac{\nabla^{\prime} V\left(\mathbf{r}^{\prime}, t-\frac{\left|\mathbf{r}-\mathbf{r}^{\prime}\right|}{a_{1}}\right)}{\left|\mathbf{r}-\mathbf{r}^{\prime}\right|} d V^{\prime},
\end{aligned}
$$

where $a_{1}^{2}=2 G(1-\nu) / \rho(1-2 \nu)$ and Eq. (3.15) has the solution

$$
\nabla x \psi=\frac{1}{4 \pi G} \int_{V^{\prime}} \frac{\nabla^{\prime} x f\left(\mathbf{r}^{\prime}, t-\frac{\left|\mathbf{r}-\mathbf{r}^{\prime}\right|}{a_{2}}\right)}{\left|\mathbf{r}-\mathbf{r}^{\prime}\right|} d V^{\prime}
$$

where $a_{2}^{2}=G / \rho$.

The solutions for $\nabla \varphi$ and $\nabla x \psi$ are the standard solutions for the wave equation with sources $\nabla T, \nabla V$, and $\nabla x f$ located at the position $r^{\prime}$.

If we choose

$$
u=a\left[\frac{\partial}{\partial s} \nabla \chi-2(1-\nu) \hat{s} \nabla^{2} \chi+(1-2 \nu) \hat{s} \frac{\rho}{G} \frac{\partial^{2} \chi}{\partial t^{2}}\right]+\nabla \varphi+\nabla x \psi
$$

such that $\varphi$ is represented by Eq. (3.16) and satisfies Eq. (3.14), $\psi$ is represented by Eq. (3.17) and satisfies Eq. (3.15), $F$ is represented by $\nabla V+\nabla x f$, and substitute into Eq. (3.4), we obtain

$$
\begin{aligned}
-2 a \hat{s}\left[(1-\nu) \nabla^{4} \chi-\frac{(1-2 \nu)}{2 G} \rho \frac{\partial^{2}}{\partial t^{2}} \nabla^{2} \chi-\frac{(1-\nu)}{G} \rho \frac{\partial^{2}}{\partial t^{2}} \nabla^{2} \chi+\frac{\rho^{2}}{2 G^{2}}(1-2 \nu) \frac{\partial^{4} \chi}{\partial t^{4}}\right] \\
+\left[\frac{2(1-\nu)}{1-2 \nu} \nabla^{2} \nabla \varphi-\frac{\rho}{G} \frac{\partial^{2}}{\partial t^{2}} \nabla \varphi-\frac{2(1+\nu)}{1-2 \nu} \alpha \nabla T-\frac{1}{G} \nabla V\right] \\
+\left[\nabla^{2}(\nabla x \psi)-\frac{\rho}{G} \frac{\partial^{2}}{\partial t^{2}}(\nabla x \psi)+\frac{1}{G} \nabla x f\right]=0
\end{aligned}
$$

The terms in the second and third brackets are identically zero, since they are identical to Eq. (3.14) and (3.15). Equation (3.19) then reduces to Eq. (3.10) which is the fourth order equation for $\chi$. We have arbitrarily chosen the constant $a$ to be $(-1 / 2 G)$.

4. Displacements, strains, and stresses. The displacements are now defined to be

$$
u_{i}=-\frac{1}{2 G} \frac{\partial}{\partial s} \frac{\partial \chi}{\partial x_{i}}+\frac{1-\nu}{G} \delta_{i, s} \nabla^{2} \chi-\frac{\rho}{2 G^{2}}(1-2 \nu) \delta_{i, s} \frac{\partial^{2} \chi}{\partial t^{2}}+\frac{\partial \varphi}{\partial x_{i}}+(\nabla x \psi)_{i} .
$$

The strains become

$$
\begin{aligned}
\epsilon_{i i}=-\frac{1}{2 G} \frac{\partial}{\partial s} & \frac{\partial^{2} \chi}{\partial x_{i} \partial x_{i}}+\frac{1-\nu}{2 G} \delta_{i, s} \frac{\partial}{\partial x_{i}} \nabla^{2} \chi+\frac{1-\nu}{2 G} \delta_{i, s} \frac{\partial}{\partial x_{i}} \nabla^{2} \chi \\
& -\frac{\rho}{4 G^{2}}(1-2 \nu) \delta_{i, s} \frac{\partial}{\partial x_{i}} \frac{\partial^{2} \chi}{\partial t^{2}}-\frac{\rho}{4 G^{2}}(1-2 \nu) \delta_{i, s} \frac{\partial}{\partial x_{i}} \frac{\partial^{2} \chi}{\partial t^{2}} \\
& +\frac{1}{2} \frac{\partial}{\partial x_{i}}\left(\frac{\partial \varphi}{\partial x_{i}}\right)+\frac{1}{2} \frac{\partial}{\partial x_{i}}\left(\frac{\partial \varphi}{\partial x_{i}}\right)+\frac{1}{2} \frac{\partial}{\partial x_{i}}(\nabla x \psi)_{i}+\frac{1}{2} \frac{\partial}{\partial x_{i}}(\nabla x \psi)_{i}
\end{aligned}
$$

The stresses are found from the Duhamel-Neumann relations to be 


$$
\begin{aligned}
\sigma_{i j}=\nu \delta_{i j} & \frac{\partial}{\partial s} \nabla^{2} \chi-\frac{\partial}{\partial s} \frac{\partial^{2} \chi}{\partial x_{i} \partial x_{i}}+(1+\nu) \delta_{i, s} \frac{\partial}{\partial x_{i}} \nabla^{2} \chi \\
& +(1-\nu) \delta_{j, s} \frac{\partial}{\partial x_{i}} \nabla^{2} \chi-\frac{\nu \rho}{G} \delta_{i j} \frac{\partial}{\partial s} \frac{\partial^{2} \chi}{\partial t^{2}}-\frac{\rho}{2 G}(1-2 \nu) \delta_{i, s} \frac{\partial}{\partial x_{i}} \frac{\partial^{2} \chi}{\partial t^{2}} \\
& -\frac{\rho}{2 G}(1-2 \nu) \delta_{j, s} \frac{\partial}{\partial x_{i}} \frac{\partial^{2} \chi}{\partial t^{2}}+\frac{2 \nu G}{1-2 \nu} \delta_{i j} \nabla^{2} \varphi+G \frac{\partial}{\partial x_{i}}\left(\frac{\partial \varphi}{\partial x_{j}}\right) \\
& +G \frac{\partial}{\partial x_{i}}\left(\frac{\partial \varphi}{\partial x_{i}}\right)+G \frac{\partial}{\partial x_{i}}(\nabla x \psi)_{i}+G \frac{\partial}{\partial x_{i}}(\nabla x \psi)_{i}-\frac{2 G(1+\nu)}{1-2 \nu} \delta_{i j} \alpha T .
\end{aligned}
$$

The $\hat{s}$ axis is chosen to be the axis of symmetry. In the case of bodies of revolution the axis of symmetry is generally chosen to be the $z$ axis. In the absence of symmetry, we shall choose the $\hat{s}$ axis to be the $z$ axis. The quantities $\partial \varphi / \partial x_{i}$ and $(\nabla x \psi)_{i}$ are given by Eqs. (3.16) and (3.17) respectively.

No boundary conditions have been specified in the solution to the equilibrium and compatibility equations. Any combination of boundary conditions on the displacements and stress components can be specified since they are derived from the same scalar stress function. The boundary conditions must, of course, be consistent with the equilibrium and compatibility conditions.

The only assumptions made are that the displacements are small, i.e., the quadratic terms in the strain can be neglected, and that the surfaces are simply connected. For bodies which have holes, double surfaces, etc., it will be necessary to break up these bodies into simple geometries and apply connecting boundary conditions where connections exist.

5. Appendix. Stress Components in Curvilinear Coordinates. It would be instructive to see how the stresses and displacements appear in an other than Cartesian coordinate system. To do this let us write Eqs. (4.1) and (4.3) in dyadic form. Thus

$$
\begin{aligned}
\mathbf{u}=-\frac{1}{2 G} \nabla \frac{\partial \chi}{\partial s}+\frac{1-\nu}{G} & \hat{s} \nabla^{2} \chi-\frac{\rho}{2 G^{2}}(1-2 \nu) \hat{s} \frac{\partial^{2} \chi}{\partial t^{2}}+\mathbf{w} \\
\hat{\sigma}=\nu \hat{I} \frac{\partial}{\partial s} \nabla^{2} \chi-\nabla \nabla \frac{\partial \chi}{\partial s} & +(1-\nu) \hat{s}\left(\nabla \nabla^{2} \chi\right)+(1-\nu)\left(\nabla \nabla^{2} \chi\right) \hat{s}-\frac{\nu \rho}{G} \hat{I} \frac{\partial}{\partial s} \frac{\partial^{2} \chi}{\partial t^{2}} \\
& -\frac{\rho}{2 G}(1-2 \nu) \hat{s} \frac{\partial^{2}}{\partial t^{2}}(\nabla \chi)-\frac{\rho}{2 G}(1-2 \nu) \frac{\partial^{2}}{\partial t^{2}}(\nabla \chi) \hat{s} \\
& +\frac{2 \nu G}{1-2 \nu} I \nabla^{2} \Phi+G(\nabla w+w \nabla)-\frac{2 G(1+\nu)}{1-2 \nu} \hat{I} \alpha T
\end{aligned}
$$

where $I$ is the dyadic $\hat{\imath} \hat{\imath}+\hat{\jmath} \hat{\jmath}+\hat{k} \hat{k}$ and $\mathrm{w}=\nabla \Phi+\nabla x \psi$.

For the cylinder the symmetric axis is the $z$ axis and the unit dyadic is $I=\hat{r} \hat{r}+\hat{\theta} \hat{\theta}+$ $\hat{k} \hat{k}$. The displacements are as follows:

$$
\begin{aligned}
& u_{r}=-\frac{1}{2 G} \frac{\partial^{2} \chi}{\partial r \partial z}+w_{r} \\
& u_{\theta}=-\frac{1}{2 G} \frac{1}{r} \frac{\partial^{2} \chi}{\partial \theta \partial z}+w_{\theta} \\
& u_{z}=-\frac{1}{2 G} \frac{\partial^{2} \chi}{\partial z^{2}}+\frac{1-\nu}{G} \nabla^{2} \chi-\frac{\rho}{2 G^{2}}(1-2 \nu) \frac{\partial^{2} \chi}{\partial t^{2}}+w_{z}
\end{aligned}
$$


The stresses are as follows:

$$
\begin{gathered}
\sigma_{r r}=\frac{\partial}{\partial z}\left(\nu \nabla^{2} \chi-\frac{\partial^{2} \chi}{\partial r^{2}}-\frac{\nu \rho}{G} \frac{\partial^{2} \chi}{\partial t^{2}}\right)+\frac{2 \nu G}{1-2 \nu} \nabla^{2} \Phi+2 G \frac{\partial w_{r}}{\partial r}-2 G \frac{(1+\nu)}{1-2 \nu} \alpha T, \\
\sigma_{\theta \theta}=\frac{\partial}{\partial z}\left(\nu \nabla^{2} \chi-\frac{1}{r} \frac{\partial \chi}{\partial r}-\frac{1}{r^{2}} \frac{\partial^{2} \chi}{\partial \theta^{2}}-\frac{\nu \rho}{G} \frac{\partial^{2} \chi}{\partial t^{2}}\right) \\
\quad+\frac{2 \nu G}{1-2 \nu} \nabla^{2} \Phi+\frac{2 G}{r}\left(w_{r}+\frac{\partial w_{\theta}}{\partial \theta}\right)-2 G \frac{(1+\nu)}{1-2 \nu} \alpha T, \\
\sigma_{z z}=\frac{\partial}{\partial z}\left[(2-\nu) \nabla^{2} \chi-\frac{\partial^{2} \chi}{\partial z^{2}}-(1-\nu) \frac{\rho}{G} \frac{\partial^{2} \chi}{\partial t^{2}}\right] \\
\quad+\frac{2 \nu G}{1-2 \nu} \nabla^{2} \Phi+2 G \frac{\partial w_{z}}{\partial z}-2 G \frac{(1+\nu)}{1-2 \nu} \alpha T ; \\
\sigma_{r \theta}=-\frac{\partial}{\partial z}\left(\frac{1}{r} \frac{\partial^{2} \chi}{\partial r \partial \theta}-\frac{1}{r^{2}} \frac{\partial \chi}{\partial \theta}\right)+\frac{2 G}{r}\left(\frac{\partial w_{r}}{\partial \theta}+w_{\theta}\right)+2 G \frac{\partial w_{\theta}}{\partial r}, \\
\sigma_{r z}=\frac{\partial}{\partial r}\left[(1-\nu) \nabla^{2} \chi-\frac{\partial^{2} \chi}{\partial z^{2}}-\frac{\rho}{2 G}(1-2 \nu) \frac{\partial^{2} \chi}{\partial t^{2}}\right]+2 G\left(\frac{\partial w_{r}}{\partial z}+\frac{\partial w_{z}}{\partial r}\right), \\
\sigma_{\theta z}=\frac{1}{r} \frac{\partial}{\partial \theta}\left[(1-\nu) \nabla^{2} \chi-\frac{\partial^{2} \chi}{\partial z^{2}}-\frac{\rho}{2 G}(1-2 \nu) \frac{\partial^{2} \chi}{\partial t^{2}}\right]+2 G\left(\frac{\partial w_{\theta}}{\partial z}+\frac{1}{r} \frac{\partial w_{z}}{\partial \theta}\right) .
\end{gathered}
$$

In the absence of body forces and temperature fields, the displacements and stress depend only on the stress function. Further, if there is no angular and time dependence, Eq. (A.4) reduces to Timoshenko's result and the equation the stress function must satisfy is the biharmonic Eq. $\nabla^{4} \chi=0$.

For the sphere the unit dyadic is $I=\hat{r} \hat{r}+\hat{\theta} \hat{\theta}+\hat{\Phi} \hat{\Phi}$. The symmetric axis is the $z$ axis and is represented by $\hat{k}=\hat{r} \cos \theta-\hat{\theta} \sin \theta$. Furthermore $\partial / \partial s=\cos \theta(\partial / \partial r)-$ $(1 / r) \sin \theta(\partial / \partial \theta)$. The displacements become

$$
\begin{aligned}
u_{r}=-\frac{1}{2 G} \frac{\partial}{\partial r}\left(\cos \theta \frac{\partial \chi}{\partial r}-\frac{1}{r}\right. & \left.\sin \theta \frac{\partial \chi}{\partial \theta}\right) \\
& +\frac{(1-\nu)}{G} \cos \theta \nabla^{2} \chi-\frac{(1-2 \nu) \rho}{2 G^{2}} \cos \theta \frac{\partial^{2} \chi}{\partial t^{2}}+w_{r} \\
u_{\theta}=-\frac{1}{2 G} \frac{1}{r} \frac{\partial}{\partial \theta}\left(\cos \theta \frac{\partial \chi}{\partial r}-\right. & \left.\frac{1}{r} \sin \theta \frac{\partial \chi}{\partial \theta}\right) \\
& -\frac{(1-\nu)}{G} \sin \theta \nabla^{2} \chi+\frac{(1-2 \nu) \rho}{2 G^{2}} \sin \theta \frac{\partial^{2} \chi}{\partial t^{2}}+w_{\theta}
\end{aligned}
$$

$u_{\Phi}=-\frac{1}{2 G} \frac{1}{r} \sin \theta \frac{\partial}{\partial \Phi}\left(\cos \theta \frac{\partial \chi}{\partial r}-\frac{1}{r} \sin \theta \frac{\partial \chi}{\partial \theta}\right)+w_{\Phi}$.

The stresses are

$$
\begin{aligned}
\sigma_{r r} & =\cos \theta \frac{\partial}{\partial r}\left[(2-\nu) \nabla^{2} \chi-\frac{\rho}{G}(1-\nu) \frac{\partial^{2} \chi}{\partial t^{2}}\right]-\frac{1}{r} \sin \theta \frac{\partial}{\partial \theta}\left[\nu \nabla^{2} \chi-\frac{\rho \nu}{G} \frac{\partial^{2} \chi}{\partial t^{2}}\right] \\
& -\frac{\partial^{2}}{\partial r^{2}}\left[\cos \theta \frac{\partial \chi}{\partial r}-\frac{1}{r} \sin \theta \frac{\partial \chi}{\partial \theta}\right]+\frac{2 \nu G}{1-2 \nu} \nabla^{2} \Phi+2 G \frac{\partial w_{r}}{\partial r}-2 G \frac{(1+\nu)}{1-2 \nu} \alpha T,
\end{aligned}
$$


$\sigma_{\theta \theta}=\cos \theta \frac{\partial}{\partial r}\left[\nu \nabla^{2} \chi-\frac{\rho \nu}{G} \frac{\partial^{2} \chi}{\partial t^{2}}\right]-\frac{1}{r} \sin \theta \frac{\partial}{\partial \theta}\left[(2-\nu) \nabla^{2} \chi-\frac{\rho}{G}(1-\nu) \frac{\partial^{2} \chi}{\partial t^{2}}\right]$

$$
\begin{aligned}
& -\left(\frac{1}{r} \frac{\partial}{\partial r}+\frac{1}{r^{2}} \frac{\partial^{2}}{\partial \theta^{2}}\right)\left[\cos \theta \frac{\partial \chi}{\partial r}-\frac{1}{r} \sin \theta \frac{\partial \chi}{\partial \theta}\right]+\frac{2 \nu G}{1+2 \nu} \nabla^{2} \Phi \\
& +\frac{2 G}{r}\left[w_{r}+\frac{\partial w_{\theta}}{\partial \theta}\right]-2 G \frac{(1+\nu)}{1-2 \nu} \alpha T
\end{aligned}
$$

$\sigma_{\Phi \Phi}=\cos \theta \frac{\partial}{\partial r}\left[\nu \nabla^{2} \chi-\frac{\rho \nu}{G} \frac{\partial^{2} \chi}{\partial t^{2}}\right]-\frac{1}{r} \sin \theta \frac{\partial}{\partial \theta}\left[\nu \nabla^{2} \chi-\frac{\rho \nu}{G} \frac{\partial^{2} \chi}{\partial t^{2}}\right]$

$-\left(\frac{1}{r} \frac{\partial}{\partial r}+\frac{1}{r^{2}} \cot \theta \frac{\partial}{\partial \theta}+\frac{1}{r^{2}} \csc ^{2} \theta \frac{\partial^{2}}{\partial \Phi^{2}}\right)\left(\cos \theta \frac{\partial \chi}{\partial r}-\frac{1}{r} \sin \theta \frac{\partial \chi}{\partial \theta}\right)$

$+\frac{2 G}{r}\left(w_{r}+\cot \theta w_{\theta}+\csc \theta \frac{\partial w_{\Phi}}{\partial \Phi}\right)-2 G \frac{(1+\nu)}{1-2 \nu} \alpha T$

$\sigma_{r \theta}=-\sin \theta \frac{\partial}{\partial r}\left[(1-\nu) \nabla^{2} \chi-\frac{\rho}{G}(1-2 \nu) \frac{\partial^{2} \chi}{\partial t^{2}}\right]$

$$
\begin{aligned}
& +\frac{1}{r} \cos \theta \frac{\partial}{\partial \theta}\left[(1-\nu) \nabla^{2} \chi-\frac{\rho}{G}(1-2 \nu) \frac{\partial^{2} \chi}{\partial t^{2}}\right] \\
& -\frac{\partial^{2}}{\partial r \partial \theta}\left[\frac{1}{r} \cos \theta \frac{\partial \chi}{\partial r}-\frac{1}{r^{2}} \sin \theta \frac{\partial \chi}{\partial \theta}\right]+2 G\left[\frac{1}{r} \frac{\partial w_{r}}{\partial \theta}+r \frac{\partial}{\partial r}\left(\frac{w_{\theta}}{r}\right)\right],
\end{aligned}
$$

$\sigma_{r \Phi}=-\csc \theta \frac{\partial^{2}}{\partial r \partial \Phi}\left[\frac{1}{r} \cos \theta \frac{\partial \chi}{\partial r}-\frac{1}{r^{2}} \sin \theta \frac{\partial \chi}{\partial \theta}\right]+2 G\left[\frac{1}{r} \csc ^{2} \theta \frac{\partial w_{r}}{\partial \Phi}+r \frac{\partial}{\partial r}\left(\frac{w_{\Phi}}{r}\right)\right]$

$\sigma_{\theta \Phi}=-\frac{1}{r^{2}} \csc \theta \frac{\partial^{2}}{\partial \theta \partial \Phi}\left(\cos \theta \frac{\partial \chi}{\partial r}-\frac{1}{r} \sin \theta \frac{\partial \chi}{\partial \theta}\right)$

$$
\begin{aligned}
& -\frac{1}{r^{2}}\left(\csc \theta \cot \theta \frac{\partial}{\partial \Phi}\right)\left(\cos \theta \frac{\partial \chi}{\partial r}-\frac{1}{r} \sin \theta \frac{\partial \chi}{\partial \theta}\right) \\
& +2 G\left[\frac{1}{r \sin \theta} \frac{\partial w_{\theta}}{\partial \Phi}+\frac{\sin \theta}{r} \frac{\partial}{\partial \theta}\left(\csc \theta w_{\Phi}\right)\right] .
\end{aligned}
$$

